\title{
Physics and chemistry of hot molecular cores
}

\author{
H. Beuther \\ Max-Planck-Institute for Astronomy, Königstuhl 17, 69117 Heidelberg, Germany \\ email: beuther@mpia.de
}

\begin{abstract}
Young massive star-forming regions are known to produce hot molecular gas cores (HMCs) with a rich chemistry. While this chemistry is interesting in itself, it also allows to investigate important physical parameters. I will present recent results obtained with highangular-resolution interferometers disentangling the small-scale structure and complexity of various molecular gas components. Early attempts to develop a chemical evolutionary sequence are discussed. Furthermore, I will outline the difficulty to isolate the right molecular lines capable to unambiguously trace potential massive accretion disks.
\end{abstract}

Keywords. accretion disks, astrochemistry, techniques: interferometers, stars: early-type, ISM: kinematics and dynamics, ISM: molecules

\section{Introduction}

Hot molecular cores (HMCs) are characterized by gas temperatures exceeding $100 \mathrm{~K}$ and a rich chemistry observable in molecular line emission at (sub)mm wavelength. These HMCs are considered to represent an early evolutionary stage in high-mass star formation where the protostars are still actively accreting and ultracompact HiI regions have not yet formed (e.g., Kurtz et al. 2000; Beuther et al. 2006). Single-dish observations toward HMCs revealed stunning molecular line forests, but they were not capable to spatially resolve the various molecular components (e.g., Blake et al. 1987; Schilke et al. 1997; Hatchell et al. 1998). Only interferometric high-spatial-resolution observations resolve the spatial complexity in more detail (e.g., Wright et al. 1996; Blake et al. 1996; Wyrowski et al. 1999). For the closest and best known HMC Orion-KL, recent observations with the Submillimeter Array (SMA) dissected its molecular components showing significant spatial differences between, e.g, $\mathrm{SiO}$, oxygen-bearing species like $\mathrm{CH}_{3} \mathrm{OH}$, nitrogen-bearing species like $\mathrm{CH}_{3} \mathrm{CN}$ or sulphur-bearing species like $\mathrm{SO}_{2}$ (Beuther et al. 2005a). In the following, I will present recent SMA results toward the HMC in G29.96 as well as a molecular comparison of high-spatial-resolution observation of various massive star-forming regions. Finally, the difficulties of identifying molecular line tracers for massive disks will be discussed.

\section{The hot molecular core G29.96}

We used the SMA to observe the well-known HMC G29.96 in a broad range of spectral line and continuum emission around $862 \mu \mathrm{m}$ (Beuther et al. in prep.). The achieved angular resolution is exceptional of the order $0.3^{\prime \prime}$ for the continuum and $0.6^{\prime \prime}$ for the line emission. The submm continuum data resolved the previously identified HMC (e.g., Cesaroni et al. 1994) into four sub-sources within a projected area of $\sim 6900(\mathrm{AU})^{2}$. These four source comprise a proto-Trapezium system, and assuming spherical symmetry one can estimate an approximate protostellar density of $2 \times 10^{5}$ protostars $/ \mathrm{pc}^{3}$.

Within the given bandpass of $4 \mathrm{GHz}$ we detected $\sim 80$ spectral lines from 18 molecular species, isotopologues or vibrational excited species, with a minor fraction of $\sim 5 \%$ of 


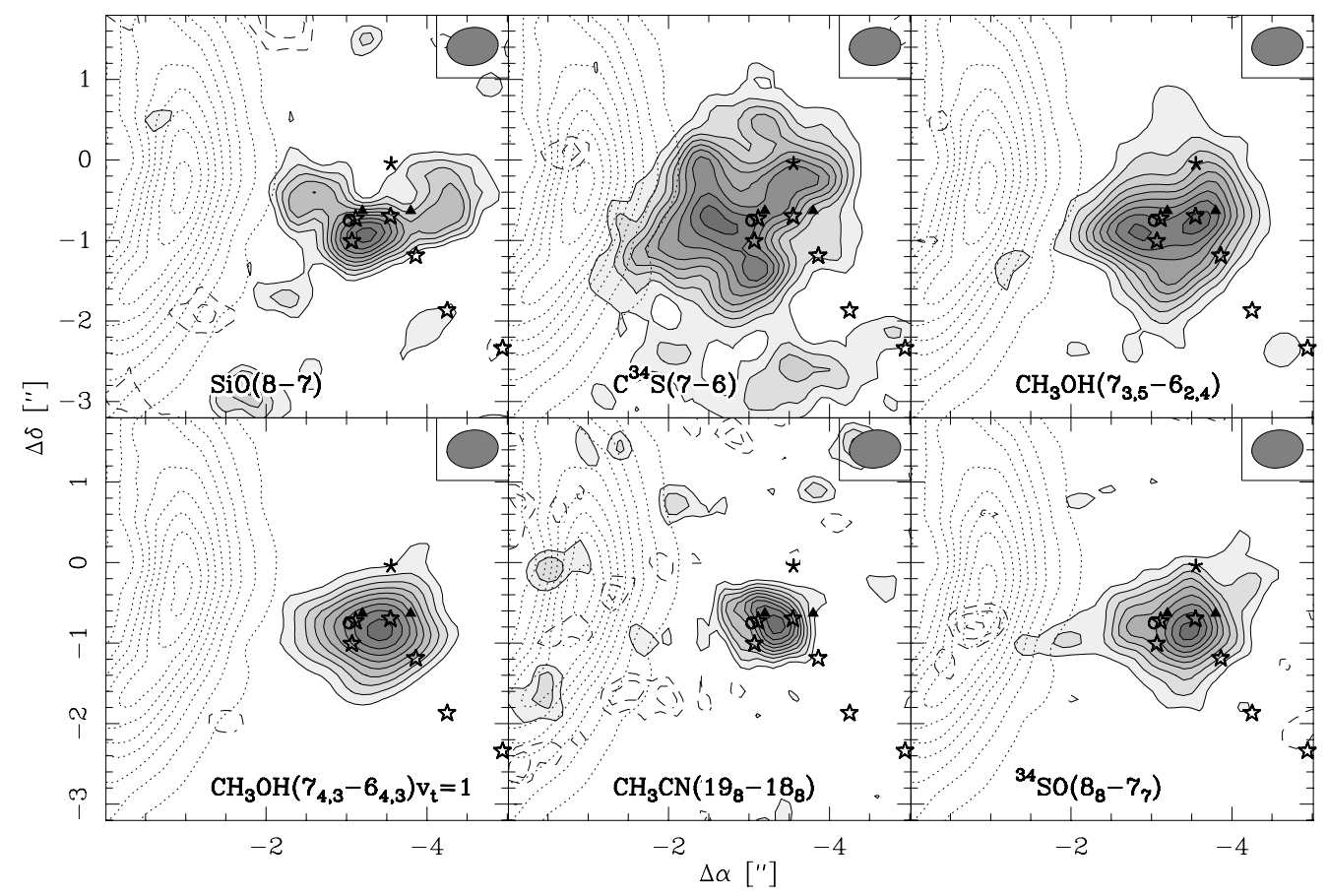

Figure 1. Spectral line maps toward the HMC G29.96 observed with the SMA (Beuther et al. in prep.). The dotted contour always show the UCHII region as observed at $\mathrm{cm}$ wavelengths (Cesaroni et al. 1994). The grey-scale with contours shows the integrated line emission from the species labeled in each panel. The stars mark submm continuum peaks identified during the same observations. The triangles and circles show $\mathrm{H}_{2} \mathrm{O}$ and $\mathrm{H}_{2} \mathrm{CO}$ maser emission, respectively (Hofner \& Churchwell 1996; Hoffman et al. 2003), the asterisks mark the mid-infrared peak from De Buizer et al. (2002), and the synthesized beam is shown at the top-right of each panel.

unidentified lines. The range of excitation temperatures for the set of lines varies between 40 and $750 \mathrm{~K}$, hence we are capable to study the cold and the warm gas at the same time. Figure 1 presents a compilation of integrated emission maps from a few representative species, and one can already discern from these maps the spatial complexity of the region. A detailed analysis of the whole dataset will be published shortly (Beuther et al. in prep.), here I only want to highlight a few characteristics:

- $\mathrm{SiO}$ shows some extended emission. Analyzing the spectral data-cube, we identify at least one, potentially two molecular outflows.

- $\mathrm{C}^{34} \mathrm{~S}$ is weak toward the HMC center and the four submm peaks, but it shows strong emission at the edge of the HMC and its interface to the UCHII region. This may be interpreted as chemical evolution: Early-on, at temperatures of the order $30 \mathrm{~K}$ CS gets released from the dust grain and the $\mathrm{C}^{34} \mathrm{~S}$ map should have appeared centrally peaked at that time. However, when the $\mathrm{HMC}$ heats up to $\geqslant 100 \mathrm{~K}, \mathrm{H}_{2} \mathrm{O}$ is released from the grains, this dissociates to $\mathrm{OH}$, and the $\mathrm{OH}$ reacts with the $\mathrm{S}$ to form $\mathrm{SO}$, which is centrally peaked then (Fig. 1). This leaves significant $\mathrm{C}^{34} \mathrm{~S}$ only at the edges of the HMC.

- No spectral line shows the same morphology as the submm continuum emission (even after smoothing the continuum to the spatial resolution of the line emission). Hence none traces unambiguously the protostellar condensations. In addition to outflow contributions and chemistry effects two other processes are considered to be important for that. On the one hand, many spectral lines are optically thick and therefore only trace the outer envelope of the region without penetrating toward the central protostellar cores. On 
the other hand, we are likely suffering from confusion because the molecules are not exclusively found in the central protostellar cores but also in the surrounding envelope. Disentangling these components is a difficult task.

- Of the many molecular lines, only a single one exhibits a coherent velocity structure with a velocity gradient perpendicular to the main outflow. Since this structure comprises three of the submm peaks it likely is a larger-scale rotating toroid which may (or may not) harbor accretion disks closer to the protostellar condensations. For the difficulties of massive disk studies see $\S 4$.

\section{Toward a chemical evolutionary sequence}

With the long-term goal in mind to establish chemical sequences - in an evolutionary sense as well as with varying luminosity - over the last few years we observed four massive star-forming regions with the SMA in exactly the same spectral setup around $862 \mu \mathrm{m}$ as used originally for the Orion-KL observations (Beuther et al. 2005a). These four regions comprise a range of luminosities between $10^{3.8} \mathrm{~L}_{\odot}$ and $10^{5} \mathrm{~L}_{\odot}$, and they cover different evolutionary stages from young pre-HMCs to typical HMCs (Orion-KL: HMC, $L \sim 10^{5} \mathrm{~L}_{\odot}, D \sim 0.45 \mathrm{kpc}$; G29.96: HMC, $L \sim 9 \times 10^{4} \mathrm{~L}_{\odot}, D \sim 6 \mathrm{kpc}$; IRAS 23151 , pre-HMC, $L \sim 10^{5} \mathrm{~L}_{\odot}, D \sim 5.7 \mathrm{kpc}$; IRAS 05358: pre-HMC, $L \sim 10^{3.8} \mathrm{~L}_{\odot}, D \sim 1.8 \mathrm{kpc}$ ). Smoothing all datasets to the same linear spatial resolution, we are now capable to start comparing these different regions. Figure 2 presents typical spectra extracted toward the HMC G29.96 and the pre-HMC IRAS 23151 (Beuther et al. in prep.).
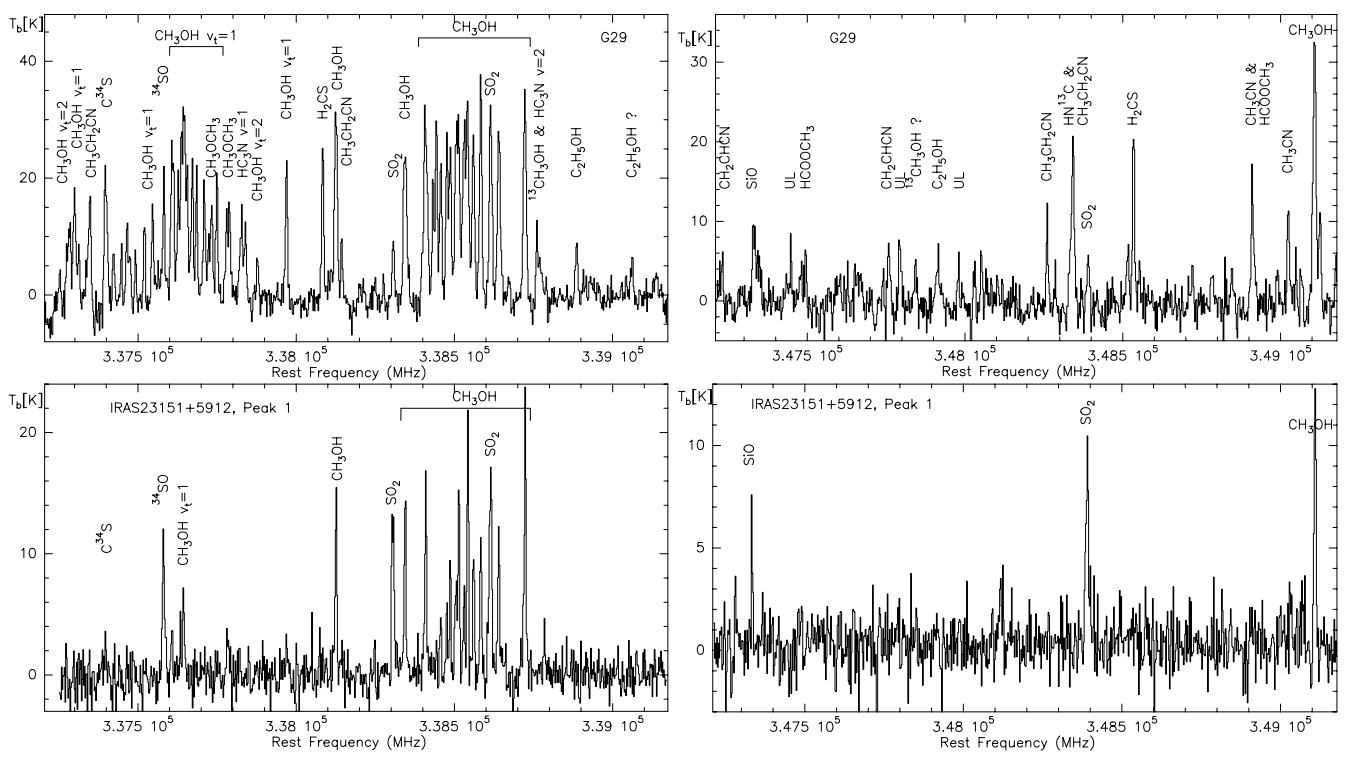

Figure 2. SMA spectra extracted from the final data-cubes toward two massive star-forming regions (G29.96 top row \& IRAS $23151+5912$ bottom row). The spectral resolution in all spectra is $2 \mathrm{~km} / \mathrm{s}$. The left and right column show the lower and upper sideband data, respectively.

A detailed comparison between the four sources will be given in a forthcoming paper (Beuther et al. in prep.), here I just outline a few differences in a qualitative manner.

- The HMCs show far more molecular lines than the pre-HMCs. Orion-KL and G29.96 appear similar indicating that the nature of the two sources may be similar as well. Regarding the two pre-HMCs, the higher luminosity one (IRAS 23151) shows still more 
lines than the lower-luminosity source (IRAS 05358). Since IRAS 05358 is approximately three times closer to us than IRAS 23151, this is not a sensitivity issue but it is likely due to the different luminosity objects forming at the core centers.

- The ground-state $\mathrm{CH}_{3} \mathrm{OH}$ lines are detected toward all four sources. However, the vibrational-torsional excited $\mathrm{CH}_{3} \mathrm{OH}$ are only strongly detected toward the HMCs OrionKL and G29.96. Independent of the luminosity, the pre-HMCs exhibit only one $\mathrm{CH}_{3} \mathrm{OH}$ $v_{t}=1$ line, which can easily be explained by the lower average temperatures of the pre-HMCs.

- A more subtle difference can be discerned by comparing the $\mathrm{SO}_{2}$ and the $\mathrm{HN}^{13} \mathrm{C}$ lines near $348.35 \mathrm{GHz}$ (in the upper sideband). While the $\mathrm{SO}_{2}$ line is found toward all four sources, the $\mathrm{HN}^{13} \mathrm{C}$ is strongly detected toward the $\mathrm{HMCs}$, but it is not found toward the pre-HMCs. In the framework of warming up HMCs, this indicates that nitrogen-bearing molecules are either released from the grains only at higher temperatures, or they are daughter molecules which need some time during the warm-up phase to be produced in gas-phase chemistry networks. In both cases, such molecules are expected to be found not much prior to the formation of a detectable HMC.

\section{Identifying molecules for massive disk studies}

There exists ample, however indirect evidence for the existence of massive disks in high-mass star formation (e.g., Cesaroni et al. 2006; Beuther et al. 2006). Theorists predict that massive accretion disks have to exist (e.g., Jijina \& Adams 1996; Yorke \& Sonnhalter 2002; Krumholz 2006), and the observations of collimated jet-like molecular outflows from at least B0 stars indicate the presence of underlying accretion disks as well (e.g., Beuther \& Shepherd 2005; Arce et al. 2006). However, we have not found much observational evidence for massive accretion disks, not to speak that we have not characterized them properly yet (Cesaroni et al. 2006). The best known example is the disk in IRAS 20126+4104 which even shows a Keplerian velocity profile, but the mass of the central object is only $\sim 7 \mathrm{M}_{\odot}$, and it is probably still in its accretion phase (Cesaroni et al. 2005). There are more sources observed where we find rotational signatures in the central cores perpendicular to the molecular outflows (see Figure 3 for a small compilation), however, the velocity structure is not Keplerian or they are that large (potentially comprising several sub-sources) that they rather resemble larger-scale rotating toroids than typical accretion disks (e.g., Cesaroni et al. 2006; Keto \& Wood 2006). Maybe these sources harbor genuine accretion disks at their very centers.

A major observational problem arises because it is difficult to disentangle the spectral line distributions from the various gas components (mainly core-disk, envelope and outflow). Some previously believed good disk-tracers have been shown to be strongly influenced by the molecular outflows (e.g., CN Beuther et al. 2004, HCN Zhang priv. comm.). Probably even more difficult, the expected central disks and the close-by surrounding envelopes are both warming up quickly due to the heating of the central accreting source. Therefore, the chemical properties - and hence the molecular emission arising from both components - are similar. This way, one may have to model always both components together. Furthermore, as we have seen in the previous sections, the chemistry varies with evolution. For example, while $\mathrm{C}^{34} \mathrm{~S}$ may be a potentially good disk tracer in young pre-HMCs like IRAS 20126+4104 (Fig. 3), it obviously does not work in more evolved HMCs like G29.96 (Fig. 1). In contrast, nitrogen-bearing molecules like $\mathrm{HN}^{13} \mathrm{C}$ appear to be a good tracer of rotation in the HMC G29.96, but it remains undetectable in younger sources like IRAS 23151+5912 (Fig. 2). An additional complication arises from varying optical depths: while the $1 \mathrm{~mm}$ lines of $\mathrm{CH}_{3} \mathrm{CN}$ are good rotation tracers in some sources 


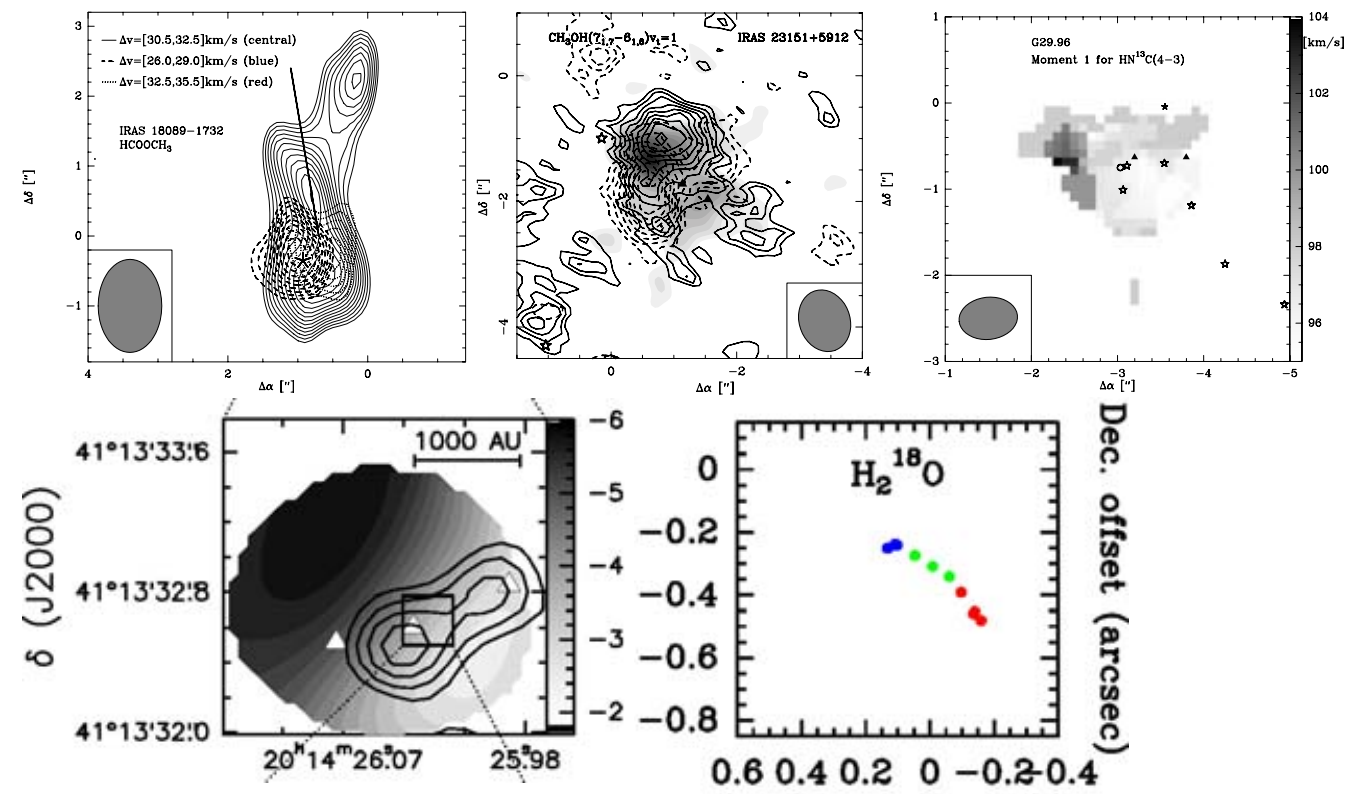

Figure 3. Examples of rotation-tracing molecules: top-left: $\mathrm{HCOOCH}_{3}$ in IRAS 18089-1732 (Beuther et al. 2005b), top-middle: $\mathrm{CH}_{3} \mathrm{OH} v_{t}=1$ in IRAS 23151+5912 (Beuther et al. in prep.), top-right: $\mathrm{HN}^{13} \mathrm{C}$ in $\mathrm{G} 29.96$ (Beuther et al. in prep.), bottom-left: $\mathrm{C}^{34} \mathrm{~S}$ (and also $\mathrm{CH}_{3} \mathrm{CN}$ ) in IRAS 20126+4104 (Cesaroni et al. 1999, 2005), bottom-right: $\mathrm{H}_{2}^{18} \mathrm{O}$ in AFGL2591 (van der Tak et al. 2006).

(Cesaroni et al. 1999; Beltrán et al. 2004), the more excited lines in the submm bands do not always show these signatures. Beuther et al. (2005b) interpreted this difference due to increased optical depth at the given high temperatures in the submm bands.

The advent of broad spectral bandpasses in new or upgraded interferometers now allows to observe many spectral lines simultaneously (Fig. 2). Thus, we can identify the best rotation-tracing molecules for individual sources after the observations without the need of strong molecular pre-selection effects. While one would like to observe a large sample of massive disk candidates in the same spectral line to study the kinematic properties as consistently as possible, this may be impossible due to the above discussed physical and chemical difficulties. However, if we are able to observe many sources systematically in a spectral setup covering the most important molecules, we can select the adequate line for each source and still investigate a larger sample in a statistically consistent manner.

\section{Conclusions and outlook}

Interferometry at (sub)mm wavelengths is the tool of choice if one wants to disentangle the chemical and physical complexity in massive star-forming regions. Only since a couple of years we are capable to spatially map a broad range of molecular lines in selected regions at high-spatial resolution.

One of the first results of these studies is that the spatial diversity of the molecules is extremely complex. For a proper understanding of the given data, it is necessary to enhance the models including physical properties like shocks, outflows, rotation and heating as well as chemical networks containing gas-phase and grain-surface reactions. Furthermore, the physical and chemical models have to be treated with state-of-the art radiative transfer codes to finally produce synthetic images which can be compared with 
the observations (e.g., Pavlyuchenkov et al. 2006). On the observational side, we need to observe larger source-samples consisting of different evolutionary stages as well as different luminosities. Observations and modeling have advanced very much over the last decade, but often the various groups did not interact enough. For a better understanding of the chemical complexity of massive star-forming regions only a concerted effort from theory, modeling and observations is likely to result in significant progress.

Massive accretion disks are considered the holy grail in high-mass star formation research. The availability of broad spectral bandpasses now allows to observe larger source samples in a less pre-selective way since it is likely that one of the observed spectral lines will trace the central rotating structure and hence allow a kinematic analysis. Utilizing the currently available (sub)mm interferometers (mainly PdBI, SMA and CARMA) as well as ALMA in the coming decade, we are expecting to reach a much better understanding of massive accretion disks and thus high-mass star formation in general.

\section{Acknowledgements}

Thanks a lot to Hendrik Linz for comments on an early draft of this paper. H.B. acknowledges financial support by the Emmy-Noether-Program of the Deutsche Forschungsgemeinschaft (DFG, grant BE2578).

\section{References}

Arce, H., Shepherd, D., Gueth, F., et al. 2006, in: B. Reipurth, D. Jewitt \& K. Keil (eds.), Protostars $\&$ Planets $V$, in press, astro-ph/0603071

Beltrán, M. T., Cesaroni, R., Neri, R., et al. 2004, ApJ 601, L187

Beuther, H., Churchwell, E., McKee, C. \& Tan, J. 2006, in: B. Reipurth, D. Jewitt \& K. Keil (eds.), Protostars $\&$ Planets $V$, in press, astro-ph/0511294

Beuther, H., Schilke, P. \& Wyrowski, F. 2004, ApJ, 615, 832

Beuther, H. \& Shepherd, D. 2005, in: M.S. Nanda Kumar, M. Tafalla \& P. Caselli (eds.) Cores to Clusters: Star Formation with Next Generation Telescopes, Ap\&SS 324, 105

Beuther, H., Zhang, Q. Greenhill, L. J., et al. 2005a, ApJ 632, 355

Beuther, H., Zhang, Q., Sridharan, T. K. \& Chen, Y. 2005b, ApJ 628, 800

Blake, G. A., Mundy, L. G., Carlstrom, J. E., et al. 1996, ApJ 472, L49

Blake, G. A., Sutton, E. C., Masson, C. R. \& Phillips, T. G. 1987, ApJ 315, 621

Cesaroni, R., Churchwell, E., Hofner, P., Walmsley, C. M. \& Kurtz, S. 1994, A\&̋A 288, 903

Cesaroni, R., Felli, M., Jenness, T., et al. 1999, A\& A 345, 949

Cesaroni, R., Galli, D., Lodato, G., Walmsley, C. \& Zhang, Q. 2006, in: B. Reipurth et al. (eds.), Protostars $\&$ Planets $V$, in press astro-ph/0603093

Cesaroni, R., Neri, R., Olmi, L., et al. 2005, A\&A 434, 1039

De Buizer, J. M., Radomski, J. T., Piña, R. K. \& Telesco, C. M. 2002, ApJ 580, 305

Hatchell, J., Thompson, M. A., Millar, T. J. \& MacDonald, G. H. 1998, A\&AS 133, 29

Hoffman, I. M., Goss, W. M., Palmer, P. \& Richards, A. M. S. 2003, ApJ 598, 1061

Hofner, P. \& Churchwell, E. 1996, A\& $A S$ 120, 283

Jijina, J. \& Adams, F. C. 1996, ApJ 462, 874

Keto, E. \& Wood, K. 2006, ApJ 637, 850

Krumholz, M. 2006, in: M. Livio \& E. Villaver (eds.) Massive Stars: From Pop III and GRBs to the Milky Way (Cambridge: Cambridge Univ.), in press, astro-ph/0607429

Kurtz, S., Cesaroni, R., Churchwell, E., Hofner, P. \& Walmsley, C. M. 2000, in: V. Mannings, A.P. Boss, S.S. Russel (eds.), Protostars and Planets IV (Tucson: Univ. of Arizona), p. 299

Pavlyuchenkov, Y., Wiebe, D., Launhardt, R. \& Henning, T. 2006, ApJ 645, 1212

Schilke, P., Groesbeck, T. D., Blake, G. A. \& Phillips, T. G. 1997, ApJS 108, 301

van der Tak, F. F. S., Walmsley, C. M., Herpin, F. \& Ceccarelli, C. 2006, A\&A 447, 1011

Wright, M. C. H., Plambeck, R. L. \& Wilner, D. J. 1996, ApJ 469, 216

Wyrowski, F., Schilke, P., Walmsley, C. M. \& Menten, K. M. 1999, ApJ 514, L43

Yorke, H. W. \& Sonnhalter, C. 2002, ApJ 569, 846 


\section{Discussion}

Elmegreen: Do you see evidence for low mass protostars forming near the high mass protostars associated with hot cores?

BEUTHER: In the case of G29.96, we can identify above the noise gas clumps down to $\sim 3 \mathrm{M}_{\odot}$, but I would expect more sensitive observations to detect even fainter sources. Furthermore, Pratap et al. (1999) detected a faint embedded cluster in that region. So, it appears, yes, there have to be low-mass stars around, it's just hard to detect and identify them at the given distances and the large gas column densities.

HANASZ: Is it possible that the hot cores are due to shock heating resulting from e.g., jet propagation?

Beuther: Probably, there exist many ways to produce hot-core like spectra. In the case of G29.96, it appears that the major hot-core chemistry driving really stems from embedded protostars, but it is less clear in the case of Orion-KL, there the hot core is $\sim 1^{\prime \prime}$ offset from the power-house source I. And there may exist other sources where shock heating could contribute as well. But my personal impression is that internal massive protostars are the most likely culprits in many hot-core regions. 